\title{
DIRECT SIMULATION OF AGGREGATION PHENOMENA
}

\author{
BERTRAND MAURY*
}

\begin{abstract}
We present here an algorithm to simulate the motion of rigid bodies subject to a non-overlapping constraint, and which tend to aggregate when they get close to each other. The motion is induced by external forces. Two types of forces are considered here: drift force induced by the action of a surrounding fluid whose motion is prescribed, and stochastic forces modelling random shocks of molecules on the surface of the bodies. The numerical approach fits into the general framework of granular flow modelling.
\end{abstract}

\section{Introduction}

We propose a numerical algorithm to simulate the motion of particles submitted to an external force (like gravity, or the force exerted by a surrounding fluid), and such that two particles put in contact tend to aggregate. What we call a particle here is a rigid sphere. The two main features of the present approach are

1. No particle is destroyed nor created. When two particles collide, they stick together, but each of them keeps its properties.

2. Collisions are not treated as events. The non-overlapping constraint is treated numerically as a set of affine conditions which are to be verified by the velocities at each time step. One may say that collisions are captured, whereas in most other methods, they are tracked.

The algorithm we propose belongs to the class of Contact Dynamics methods, introduced by Moreau [3]. In the present case, all contacts occuring during a time step are handled globally, and reactions associated to contacts are computed as a field of Lagrange multipliers associated to first order approximations of non-penetration constraints. Actual computation relies on a Uzawa algorithm, by which approximations of primal (velocity) and dual (impulses) solutions are build iteratively.

Aggregation shall be taken into account by an attraction, short-range force between particles, whose intensity dominates other forces which might tend to fragment the aggregates.

\section{Continuous problem}

2.1. Evolution equation. We consider the mechanical system of $p$ rigid spheres of radii $r_{1}, r_{2}, \ldots, r_{p}$ and masses $m_{1}, m_{2}, \ldots, m_{p}$. We introduce the configuration space

$$
Q=\left\{\mathbf{q}=\left(q_{1}, q_{2}, \cdots, q_{p}\right) \in \mathbb{R}^{3 p}\right\}
$$

and associated feasible set

$$
Q_{0}=\left\{\mathbf{q} \in Q, D_{i j}(\mathbf{q}) \geq 0 \quad \forall i, j, \quad 1 \leq i<j \leq p\right\}
$$

where

$$
D_{i j}(\mathbf{q})=\left|q_{i}-q_{j}\right|-\left(r_{i}+r_{j}\right)
$$

is the distance between spheres $i$ and $j$. Although the tangent space at any $\mathbf{q} \in Q$ can be identified to $\mathrm{Q}$ itself, we will denote by $\mathrm{T}_{Q}=\mathbb{R}^{3 p}$ this tangent space in order

*Laboratoire de Mathématique, Bât. 425, Université Paris-Sud, Campus d'Orsay, 91405 Orsay Cedex, FRANCE (Bertrand.MAURY@math.u-psud.fr). 
to distinguish velocities from positions. We introduce the outward normal cone

$$
N_{\mathbf{q}}=\left\{-\sum_{i<j} \mu_{i j} \mathbf{G}_{i j}(\mathbf{q}), \mu_{i j}=0 \text { if } D_{i j}(\mathbf{q})>0, \mu_{i j} \in \mathbb{R}^{+} \text {if } D_{i j}(\mathbf{q})=0\right\}
$$

where $\mathbf{G}_{i j}=\nabla D_{i j}$ is the gradient of the distance between two spheres $i$ and $j$. Given a pathline $t \longmapsto \mathbf{q}(t)$, we shall denote by

$$
\mathbf{u}=\left(\dot{q}_{1}, \dot{q}_{2}, \cdots, \dot{q}_{p}\right) \in \mathrm{T}_{Q}
$$

the generalized velocity vector.

We introduce a time interval $I=] 0, T$ [ and the following functional spaces:

$W^{1,1}=$ set of those $3 p$ vector-valued functions which are absolutely continuous over the time interval $I$;

$\mathrm{BV}=$ set of $3 p$ vector-valued functions with bounded variation over $I$ : $\mathrm{BV}$ is the set of functions $t \mapsto \mathbf{u}(t) \in \mathrm{T}_{Q}$, such that each component $u$ of $\mathbf{u}$ verifies

$$
\sup _{S \in \Lambda} \sum_{n=1}^{N_{S}}\left|u\left(t_{n}\right)-u\left(t_{n-1}\right)\right|<\infty
$$

where $S=\left(t_{0}, t_{1}, \ldots, t_{N_{S}}\right)$ runs over the set $\Lambda$ of increasing subdivisions of the time interval $I$;

$\mathcal{M}^{1}=$ set of $p(p-1) / 2$ vector $^{1}$ valued bounded measures on $I$ : it contains all

$$
\mu=\left(\mu_{i j}\right)_{1 \leq i<j \leq p}
$$

such that $\mu_{i j}$ is a continuous linear functional over the set $C_{0}(I)$ of continuous functions over $\bar{I}$, vanishing at 0 and $T$. For any $\mu \in \mathcal{M}^{1}$, which shall define its norm $\|\mu\|_{\mathcal{M}^{1}}$ as

$$
\|\mu\|_{\mathcal{M}^{1}}=\max _{1 \leq i<j \leq p}\left\|\mu_{i j},\right\|
$$

where $\left\|\mu_{i j}\right\|$ is defined as the supremum of $\left\langle\mu_{i j}, \varphi\right\rangle$ over the set of functions $\varphi \in C_{0}(I)$ with $L^{\infty}$-norm less than 1 .

The set of those measures which are positive will be denoted by

$$
\mathcal{M}_{+}^{1}=\left\{\mu=\left(\mu_{i j}\right)_{1 \leq i<j \leq p} \in \mathcal{M}^{1},\left\langle\mu_{i j}, \varphi\right\rangle \geq 0 \quad \forall \varphi \in C_{0}(I), \varphi \geq 0\right\} .
$$

The action of a surrounding fluid, with given velocity $\mathbf{U}(\mathbf{x}, t)$, is taken into account by adding a viscous friction term, acting on particle $i$ as

$$
-\xi_{i}\left(\mathbf{u}_{i}-\mathbf{U}\left(\mathbf{q}_{i}, t\right)\right)
$$

so that $\tau_{i}=m_{i} / \xi_{i}$ can be seen as a relaxation time. Parameter $\tau_{i}$ shall be taken equal to $6 \pi r_{i} \nu$, where $\nu$ is the viscosity of the surrounding fluid. We shall use abusively the notation $-\xi(\mathbf{u}-\mathbf{U}(\mathbf{q}, t))$ to represent the corresponding vector in $\mathbb{R}^{3 p}$.

Aggregation is integrated into the model by adding an short-range attraction force, which we define as

$$
\mathbf{F}(\mathbf{q})=-\kappa \sum_{i<j} \nabla\left(\Psi \circ D_{i j}\right)(\mathbf{q}(t))
$$

\footnotetext{
${ }^{1} p(p-1) / 2$ is the number of possible contacts.
} 
where $\Psi$ is the function

$$
\delta \longmapsto \Psi(\delta)=\kappa \tanh \left(\frac{\delta}{\varepsilon}\right) .
$$

Parameter $\varepsilon>0$ is the distance below which particles are considered in contact, and $\kappa / \varepsilon$ can be seen as a penalty parameter: it has to be set such that the latter force dominates other forces which might tend to separate particles. We shall not address here the problem of adding a stochastic term (like white noise) to the forcing terms. This will be done at the discrete level only (see section 4.2).

We may now state the problem: Given a time interval $I=] 0, T$ [ and initial conditions $\left(\mathbf{q}_{0}, \mathbf{u}_{0}\right) \in Q_{0} \times \mathrm{T}_{Q}$,

$$
\begin{gathered}
\text { Find }(\mathbf{q}, \mathbf{u}, \mu) \in W^{1,1} \times \mathrm{BV} \times \mathcal{M}_{+}^{1} \text { such that } \\
\mathbf{u}^{+}(0)=\mathbf{u}_{0} \\
\mathbf{q}(t)=\mathbf{q}_{0}+\int_{0}^{t} \mathbf{u}(s) d s \in Q_{0} \quad \forall t \in I \\
\mathbf{M} \dot{\mathbf{u}}=-\xi(\mathbf{u}-\mathbf{U}(\mathbf{q}, t))+\mathbf{F}(\mathbf{q})+\sum_{i<j} \mu_{i j} \mathbf{G}_{i j}(\mathbf{q}(t)), \\
\forall i<j \quad \operatorname{supp}\left(\mu_{i j}\right) \subset\left\{t, D_{i j}(\mathbf{q}(t))=0\right\} \\
\mathbf{u}^{+}(t)=\mathbf{u}^{-}(t)-P_{N_{\mathbf{q}}} \mathbf{u}^{-}(t) \quad \forall t \in I,
\end{gathered}
$$

where $\mathbf{M}$ is the mass matrix $\operatorname{diag}\left(m_{1}, m_{1}, m_{1}, m_{2}, m_{2}, \cdots, m_{p}, m_{p}\right)$,

$$
\mathbf{G}_{i j}=\left(\cdots, 0,-e_{i j}, 0, \cdots, 0, e_{i j}, 0, \cdots\right),
$$

and $P_{N_{\mathbf{q}}}$ is the Euclidian projection onto the closed convex cone $N_{\mathbf{q}}$. Equation (2.13) is to be understood in the sense of distributions.

REMARK 2.1. We shall disregard here problems possibly caused by "non feasible" initial conditions $\left(\mathbf{q}_{0} \in \partial Q_{0}\right.$ and $\mathbf{u}_{0} \notin V\left(\mathbf{q}_{0}\right)$, where $V$ is the set of admissible directions, polar cone of $\left.N_{\mathbf{q}_{0}}\right)$.

REMARK 2.2. Obstacles like walls or fixed bodies may be taken into account. For the sake of simplicity, we shall not introduce new notations. These obstacles can actually be handled within this framework by considering that they are "special" particles with infinite mass. Wall-sphere contacts activate a Lagrange multiplier which prevents overlapping, exactly as for sphere-sphere contacts.

2.2. Theoretical remarks. The model we propose, in its deterministic version (the external force depends explicitly on time, position, and velocity), fits into the general framework of differential measure inclusions (see Schatzman [5], or Moreau [4]). From the theoretical point of view, the critical point is uniqueness, which can be established only under strong regularity assumptions on the data: analiticity is required. Uniqueness is lost as soon as the external force is no longer analytic (see in Schatzman [5] or Ballard [1] counter-examples to uniqueness with a $C^{\infty}$ force). Note that continuity with respect to initial conditions does not hold in general, even in the case of analytic forcing terms. 


\section{Numerical scheme}

3.1. Approximation spaces. Let $h=T / N$ be the time step. We introduce the following spaces:

$$
\begin{gathered}
V_{h}=\left\{\mathbf{u}_{h}: I \longrightarrow \mathrm{T}_{Q}, \text { constant in }[(n-1) h, n h[, n=1, \ldots, N\},\right. \\
R_{h}=\left\{\mu_{h}: I \longrightarrow \mathbb{R}^{p(p-1) / 2}, \text { constant in }[(n-1) h, n h[, n=1, \ldots, N\},\right. \\
X_{h}=\left\{\mathbf{q}_{h}: I \longrightarrow Q, \text { continuous on I, affine in }[(n-1) h, n h[, n=1, \ldots, N\} .\right.
\end{gathered}
$$

For any $\mathbf{u}_{h} \in V_{h}$ we will denote by $\mathbf{u}_{h}^{n}$ its constant value in the subinterval [ $(n-$ 1) $h, n h$. Similarly, $\mathbf{q}_{h}^{n}=\mathbf{q}_{h}(n h)$, for any $\mathbf{q}_{h} \in X_{h}$. Note that any $\mathbf{u}_{h}$ is completely determined by its values $\left(\mathbf{u}_{h}^{1}, \mathbf{u}_{h}^{2}, \ldots, \mathbf{u}_{h}^{N}\right)$ and any motion $\mathbf{q}_{h}$ by $\left(\mathbf{q}_{h}^{0}, \mathbf{q}_{h}^{1}, \ldots, \mathbf{q}_{h}^{N}\right)$. The approximated contact force field is completely determined by the $N p(p-1) / 2$ values $^{2}$

$$
\mu_{i j}^{n}, \quad n=1, \ldots, N, \quad 1 \leq i<j \leq p .
$$

3.2. Time stepping. $\quad\left(\mathbf{q}_{h}, \mathbf{u}_{h}, \mu_{h}\right) \in X_{h} \times V_{h} \times R_{h}$ is built as follows

1. Initialization

$$
\left(\mathbf{q}_{h}^{0}, \mathbf{u}_{h}^{0}\right)=\left(\mathbf{q}_{0}, \mathbf{u}_{0}\right) .
$$

2. Compute $\mathbf{u}_{h}^{n+1}$ and $\left(\mu_{i j}^{n+1}\right) 1 \leq i<j \leq N$ as primal and dual solutions, respectively, of the constrained minimization problem:

$$
\min _{\mathbf{u} \in K^{n}}\left|\mathbf{M u}-\mathbf{M} \mathbf{u}_{h}^{n}+h \xi\left(\mathbf{u}_{h}^{n}-\mathbf{U}\left(\mathbf{q}_{h}^{n}, t\right)\right)-h \mathbf{F}\left(\mathbf{q}_{h}^{n}\right)\right|^{2}
$$

with

$$
K^{n}=\left\{\mathbf{u} \in \mathrm{T}_{Q}, D_{i j}\left(\mathbf{q}_{h}^{n}\right)+h \mathbf{G}_{i j}\left(\mathbf{q}_{h}^{n}\right) \cdot \mathbf{u} \geq 0\right\},
$$

so that $\mathbf{u}_{h}^{n+1}$ and the $\mu_{i j}^{n+1}$ 's are related by

$$
\mathbf{M u}_{h}^{n+1}=\mathbf{M} \mathbf{u}_{h}^{n}-h \xi\left(\mathbf{u}_{h}^{n}-\mathbf{U}\left(\mathbf{q}_{h}^{n}, t\right)\right)+h \mathbf{F}\left(\mathbf{q}_{h}^{n}\right)+h \sum_{i<j} \mu_{i j}^{n+1} \mathbf{G}_{i j}\left(\mathbf{q}_{h}^{n}\right) .
$$

REMARK 3.1. Aggregation forces are taken into account explicitly, whereas they are stiff. Such a situation usually leads to instabilities and prevents us from using large time steps. The situation here is different, because this attraction force opposes the contact reaction, which is computed implicitly. High values for this attraction force may deteriorate the convergence of the Uzawa algorithm (see next section), but the overall time stability shall not be harmed.

\footnotetext{
${ }^{2}$ In order to alleviate notations, we shall drop the dependence on $h$ in the values of the discrete reaction forces $\mu_{i j}^{n}$.
} 
3.3. Saddle-point problem. The constrained optimization problem (3.5)(3.6) which has to be solved at each time step is put into a saddle-point form. We introduce the vectors and matrices $\left(\mathbf{f}_{h}^{n+1}\right.$ stands for the sum of drift and attraction forcing terms)

$$
\begin{gathered}
\mathbf{u}=\mathbf{u}_{h}^{n+1} \in \mathbb{R}^{3 p}, \mathbf{F}=\mathbf{M} \mathbf{u}_{h}^{n}+h \mathbf{f}_{h}^{n+1} \in \mathbb{R}^{3 p}, \quad \mu=\left(\mu_{i j}^{n+1}\right)_{1 \leq i<j \leq p}, \\
\mathbf{C} \in \mathbb{R}^{(p-1) p / 2 \times 3 p} \text { such that }-\mathbf{C}^{T} \mu=h \sum_{i<j} \mu_{i j}^{n+1} \mathbf{G}_{i j}\left(\mathbf{q}_{h}^{n}\right),
\end{gathered}
$$

and $\mathbf{D}=\left(D_{i j}\left(\mathbf{q}_{h}^{n}\right)\right)_{1 \leq i<j \leq p}$. The problem can be put in the classical saddle point form

$$
\begin{gathered}
\text { Minimize } J(\mathbf{u})=\frac{1}{2}(\mathbf{M u}, \mathbf{u})-(\mathbf{F}, \mathbf{u}), \\
\text { over } K=\{\mathbf{u}, \mathbf{C u} \leq \mathbf{D}\} .
\end{gathered}
$$

The Lagrangian of the problem is

$$
\mathcal{L}(\mathbf{v}, \lambda)=\frac{1}{2}(\mathbf{M v}, \mathbf{v})-(\mathbf{F}, \mathbf{v})+(\lambda, \mathbf{C v}-\mathbf{D}),
$$

and any saddle-point of $\mathcal{L}$, i.e. any couple $(\mathbf{u}, \mu)$ verifying

$$
\sup _{\lambda \in \mathbb{R}_{+}^{(p-1) p / 2}} \mathcal{L}(\mathbf{u}, \lambda)=\mathcal{L}(\mathbf{u}, \mu)=\inf _{\mathbf{v} \in \mathbb{R}^{3 p}} \mathcal{L}(\mathbf{v}, \mu)
$$

is such that $\mathbf{u}$ minimizes $J$ over $K$.

The Uzawa algorithm (see Ciarlet [2]) consists in approximating a saddle-point $(\mathbf{u}, \mu)$ of $\mathcal{L}$ by sequences $\left(\mathbf{u}_{k}\right)$ and $\left(\mu_{k}\right)$. A step decomposes into two substeps:

1. Solve the primal problem

$$
\mathbf{M} \mathbf{u}_{k+1}=\mathbf{F}-\mathbf{C}^{T} \mu_{k} .
$$

2. Update the Lagrange multipliers field

$$
\left(\mu_{k+1}\right)_{i j}=\Pi_{+}\left(\mu_{k}+\rho\left(\mathbf{C u}_{k+1}-\mathbf{D}\right)\right),
$$

where $\Pi_{+}$is the orthogonal projection onto $\mathbb{R}_{+}^{(p-1) p / 2}$ :

$$
\mu \in \mathbb{R}^{(p-1) p / 2} \longmapsto \Pi_{+}(\mu)=\left(\max \left(\mu_{i j}, 0\right)\right)_{1 \leq i<j \leq p} .
$$

Sequence $\mathbf{u}_{k}$ is known to converge to the solution of the constrained minimization problem as soon as

$$
0<\rho<\frac{2 \alpha}{\left\|C C^{T}\right\|}
$$

where $\alpha$ is the smallest eigenvalue of the matrix M. In the present case $\alpha=\min \left(m_{i}\right)$. 


\section{Numerical experiments}

We considered slightly polydispersed populations, in order to avoid crystal-like arrangements. In all computations we considered a distribution of radii in the neighbourhood of a reference value $r$, between $0.85 r$ and $1.15 r$. The mass of a particle with radius $r_{i}$ is taken equal to $\left(r_{i} / r\right)^{3}$.

4.1. Deterministic forcing term. As a first test case we considered a given velocity field $\mathbf{U}$ which corresponds to an oscillating shear flow:

$$
\mathbf{U}(\mathbf{x}, t)=\sin (\omega t)\left(\begin{array}{c}
x_{2} \\
0
\end{array}\right) \quad \mathbf{x}=\left(x_{1}, x_{2}\right) .
$$

Figure 1 presents the particle cloud at different times, for a computation based on the following numerical values

$$
N=1000, \quad r=0.01, \tau=m / \xi=0.2, \omega=\pi, h=0.02, \quad \kappa=\varepsilon=3.10^{-3} .
$$

4.2. Stochastic forcing term. We apply now the presented technique to the situation where external forces are of the white-noise type. We consider the situation where the motion of a single particle (without any geometrical constraint) would obey a Langevin equation

$$
\mathrm{d} \mathbf{u}(t)=-\frac{1}{\tau} \mathbf{u}(t) \mathrm{dt}+\sigma \mathrm{d} \mathbf{W}(t)
$$

where $\mathrm{d} \mathbf{W}$ is a random vector, each component of which is the increment of a Wiener process. In order to integrate this model to the multi-body simulation, we propose the following algorithm: compute $\mathbf{u}_{h}^{n+1}$ as the solution of

$$
\min _{\mathbf{u} \in K^{n}}\left|\mathbf{M u}-\mathbf{M} \mathbf{u}_{h}^{n}+h \xi \mathbf{u}_{h}^{n}-\sqrt{h} \mathbf{F}\left(\mathbf{q}_{h}^{n}\right)-\sigma \sqrt{h} \mathbf{W}^{n}\right|^{2}
$$

where

$$
K^{n}=\left\{\mathbf{u} \in \mathrm{T}_{Q}, D_{i j}\left(\mathbf{q}_{h}^{n}\right)+h \mathbf{G}_{i j}\left(\mathbf{q}_{h}^{n}\right) \cdot \mathbf{u} \geq 0\right\},
$$

$\mathbf{W}^{n}$ is a random vector in $\mathbb{R}^{3 p}$, all components of which follow independent normal laws, and $\mathbf{F}$ is the aggregation force defined by (2.8). Numerical values for the computation are

$$
r=0.01, \quad \tau=m / \xi=0.2, \quad \sigma=0.5, \quad \kappa=\varepsilon=3.10^{-3} .
$$

Note that term $h \mathbf{F}$ is scaled by $1 / \sqrt{h}$ is order to ensure that aggregation dominates stochastic forcing.

Figures 2, 3, and 4 show the particle distributions obtained for various numbers of particles, at different times. Note that the set of times at which the pictures are taken differs from a case to the other: characteristic times at which particles aggregate depend strongly on the particle density.

REMARK 4.1. As it has been said previously, numerical parameter $\varepsilon$ represents the distance below which particles attract each other. Changing its value does not affect strongly the numerical results, as soon as $\kappa / \varepsilon$ remains constant. The value for $\kappa / \varepsilon$ has to be chosen large enough to prevent separation of particles which have collided ${ }^{3}$.

\footnotetext{
${ }^{3}$ This phenomenon cannot be completely avoided in the stochastic version of the algorithm, because the support of the random force is not compact.
} 

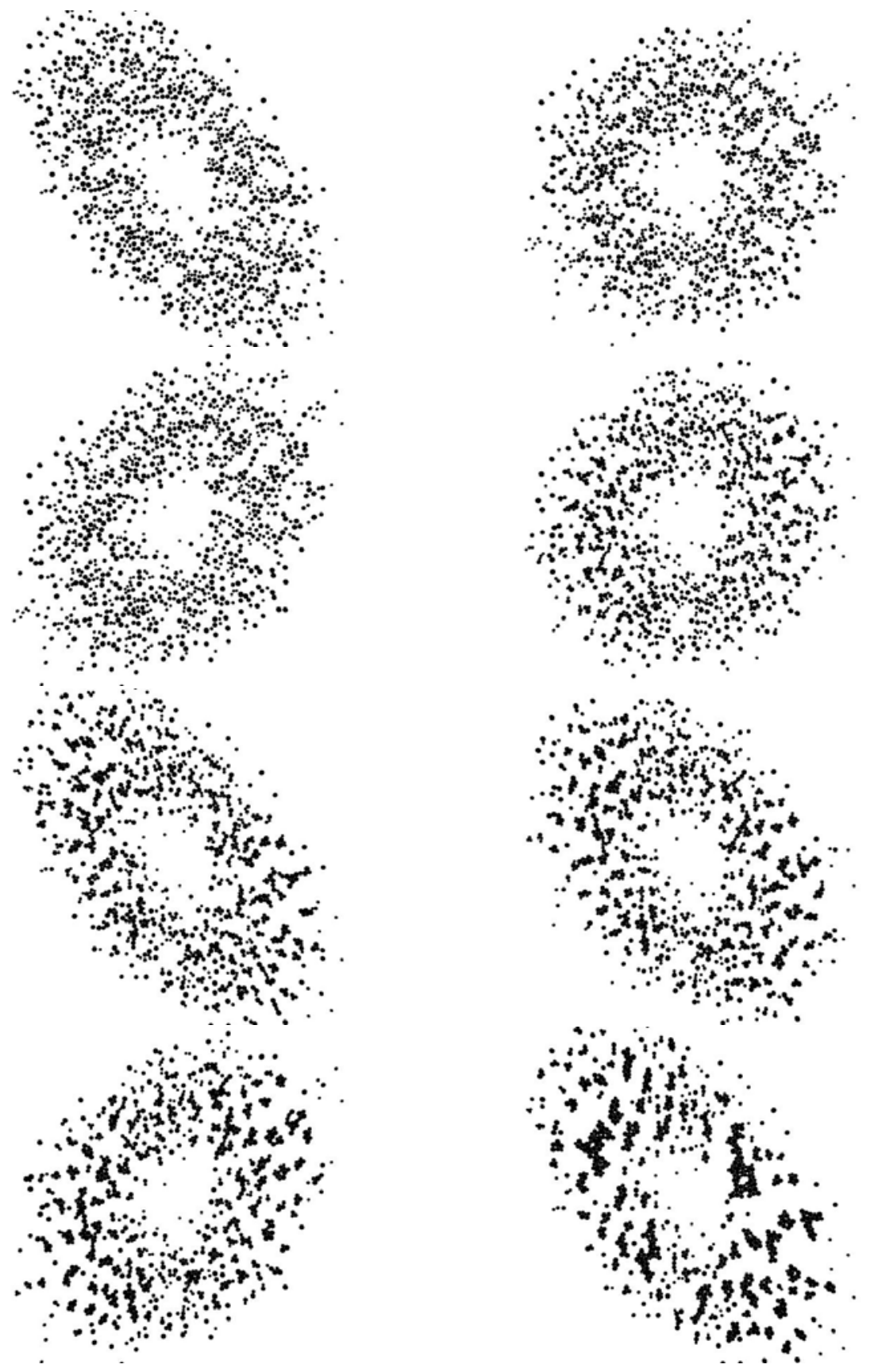

Fig. $1 . \mathrm{N}=1000$, shear flow, times $0,0.4,0.5,0.75,1.0,1.25,1.5,10$. 



Fig. 2. $\mathrm{N}=500$, times $0,1,2,3,4,5,6,7,8,9$. 



Fig. 3. $\mathrm{N}=2000$, times $0,0.4,0.6,0.8,1.0,1.2,1.8,3$. 

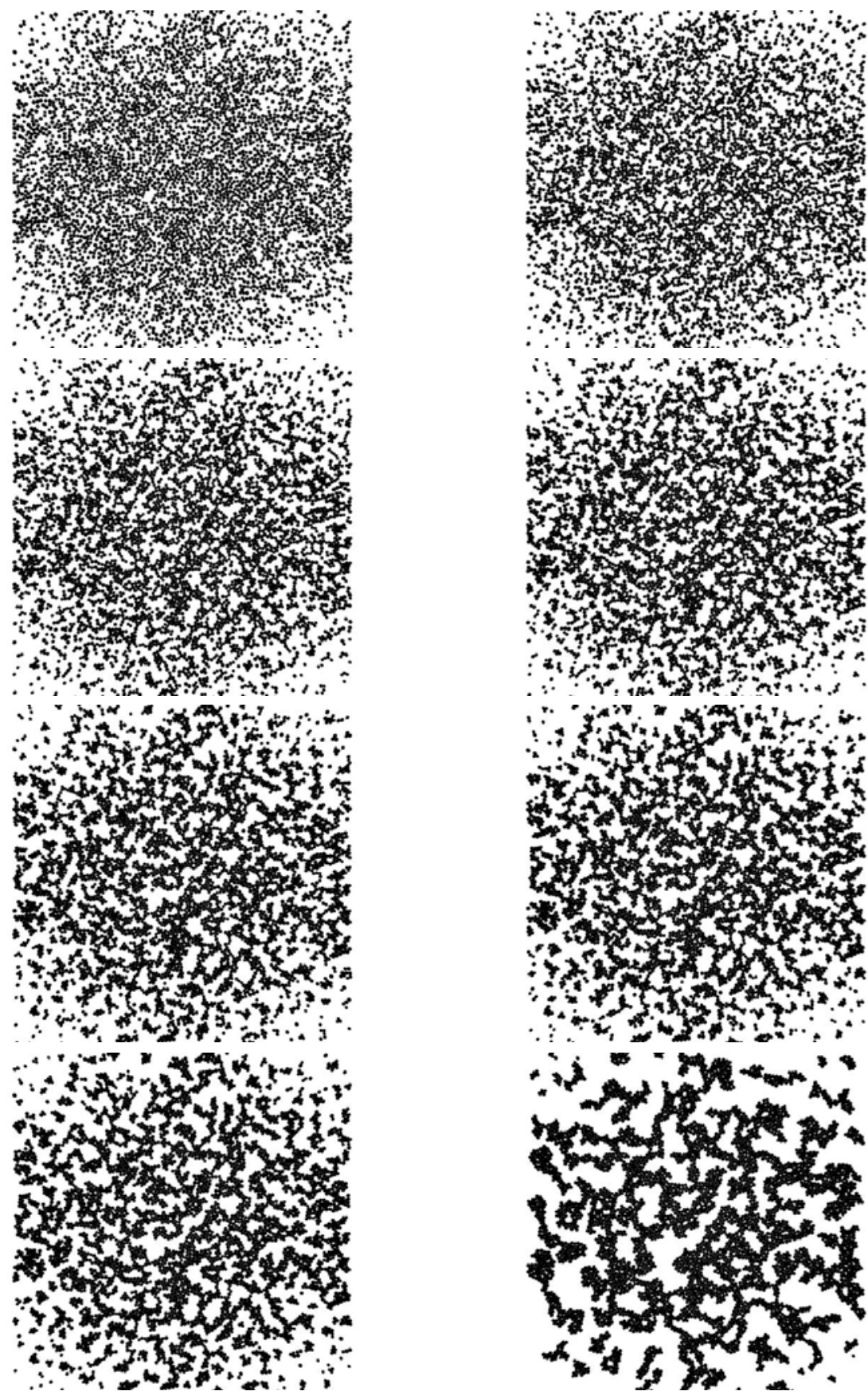

Fig. 4. $\mathrm{N}=5000$, times $0,0.1,0.15,0.2,0.3,0.4,0.5,3$.

On the other hand, increasing $\kappa / \varepsilon$ leads to higher computational costs, as it increases the attraction force between particles, which makes the saddle-point problem stiffer. 
In concrete terms, its value is set in order to dominate the forcing terms, so that most particle contacts cannot be broken.

REMARK 4.2. As soon as the computation of interparticle distances is performed in an efficient way (note that special methods should be added to the present approach in the case of highly polydisperse populations), the computational cost is linear in the number of particles. Indeed, numerical parameter $\rho$ for the Uzawa algorithm is approximately independent of the number of particles, so that the number of iterations needed to achieve a given accuracy is itself independent of this number. Only the cost of each Uzawa step varies (linearly) with the number of particles.

\section{Conclusion, future prospects}

We have proposed a basic algorithm to simulate aggregation phenomena. This algorithm makes it possible to compute directly quantities which are involved in macroscopic models, like the size distribution of the created aggregates, as functions of the parameters (mainly the initial density of single particles, and the type and intensity of external forces). This link between microscopic and macroscopic level, in which lies the real potential interest of the present approach, still has to be carried out.

From the theoretical point of view, a rigorous analysis of the continuous evolution equation in its stochastic version (white noise is added to the right-hand side) is also to be made, in order to justify the proposed model.

As for the time-discretization algorithm we propose, in the deterministic case, a proof of convergence (up to a subsequence of time steps and in the case of a single contact) will be proposed in a future paper.

\section{REFERENCES}

[1] P. Ballard, The dynamics of discrete mechanical systems with perfect unilateral constraints, Arch. Rational Mech. Anal., 154:199-274, 2000.

[2] P.G. Ciarlet, Introduction à l'Analyse Numérique Matricielle et à l'Optimisation, ed., Masson, Paris.

[3] J.J. Moreau, Some numerical methods in multibody dynamics: application to granular materials, Eur. J. mech., A/Solids, 13(4-suppl):93-114, 1994.

[4] J.J. Moreau, Bounded Variation in Time, in Topics in Non-smooth mechanics (J.J. Moreau, P.D. Panangiatopoulos, G. Strang), Birkhäuser Verlag, Basel-Boston-Berlin, 1-74, 1988.

[5] M. Schatzmann, A class of nonlinear differential equations of second order in time, Nonlinear Analysis, Theory, Methods \& Applications, 2:355-373, 1978 